\title{
Supporting Information \\ Biomaterial-Mediated Presentation of Jagged-1 Mimetic Ligand Enhances \\ Cellular Activation of Notch Signaling and Bone Regeneration
}

\author{
Yingrui Deng ${ }^{1, a}$, Rui Li ${ }^{2, a}$, Haixing Wang ${ }^{3, a}$, Boguang Yang ${ }^{1}$, Peng Shi' ${ }^{4,5,6}$, Yuan Zhang, ${ }^{4,5,6}$ \\ , Qiang Yang ${ }^{7, *}$, Gang $\mathrm{Li}^{3{ }^{3, *} \text {, Liming Bian }}{ }^{4,5,6^{*}}$ \\ ${ }^{1}$ Department of Biomedical Engineering, The Chinese University of Hong Kong, Sha Tin, New \\ Territories 999077, Hong Kong, P. R. China. \\ ${ }^{2}$ Department of Mechanical and Aerospace Engineering, Tandon School of Engineering, New \\ York University, Brooklyn, NY 11201, USA \\ ${ }^{3}$ Department of Orthopaedics and Traumatology, Faculty of Medicine, The Chinese University \\ of Hong Kong, Sha Tin, New Territories 999077, Hong Kong, P. R. China. \\ ${ }^{4}$ School of Biomedical Sciences and Engineering, Guangzhou International Campus, South \\ China University of Technology, Guangzhou 511442, P. R. China. \\ ${ }^{5}$ National Engineering Research Center for Tissue Restoration and Reconstruction, South \\ China University of Technology, Guangzhou 510006, P. R. China. \\ ${ }^{6}$ Key Laboratory of Biomedical Materials and Engineering of the Ministry of Education, South \\ China University of Technology, Guangzhou 510006, P. R. China. \\ ${ }^{7}$ Department of spine surgery, Tianjin Hospital, Tianjin University, Tianjin 300211, P.R. China. \\ ${ }^{*}$ Corresponding authors: \\ Prof. Liming Bian: bianlm@scut.edu.cn \\ Prof. Gang Li: gangli@cuhk.edu.hk \\ Prof. Qiang Yang: yangqiang1980@126.com \\ ${ }^{a}$ These authors contributed equally to this work.
}



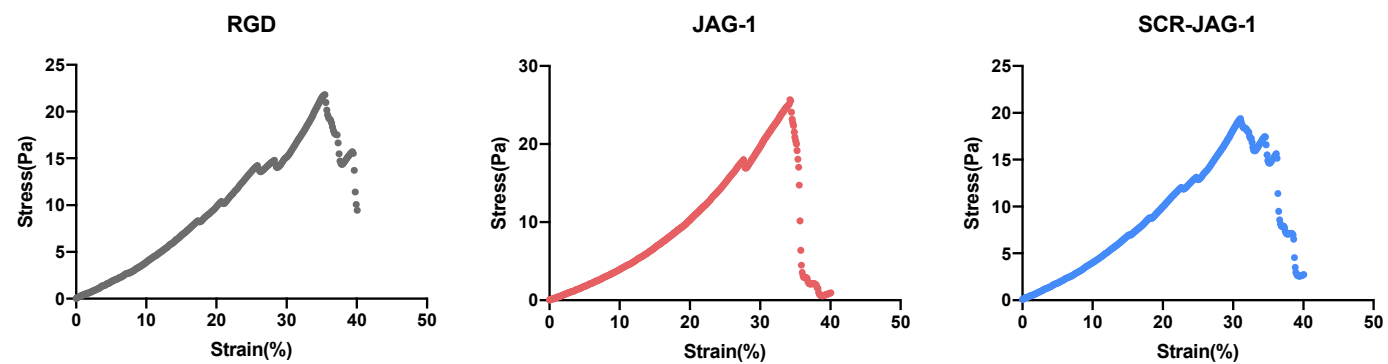

Figure S1: Strain-stress curves of RGD, JAG-1 and SCR-JAG-1 porous hydrogels. 

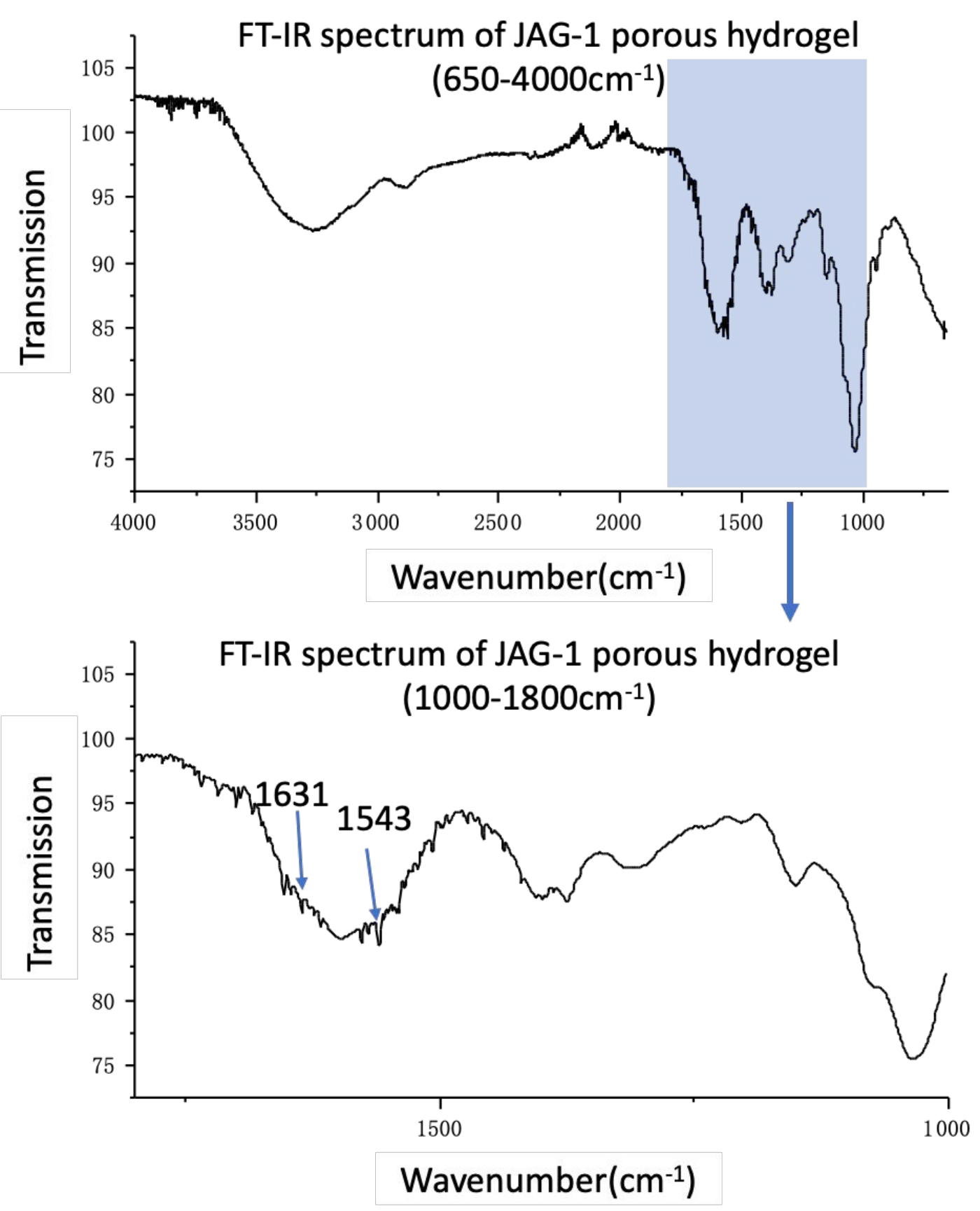

Figure S2: The FT-IR spectra of JAG-1 porous hydrogel. The presence of bands at around $1635 \mathrm{~cm}$ and $1543 \mathrm{~cm}$ in the FT-IR spectra indicate successful conjugation of peptides to the hydrogel..$^{1,2}$ 


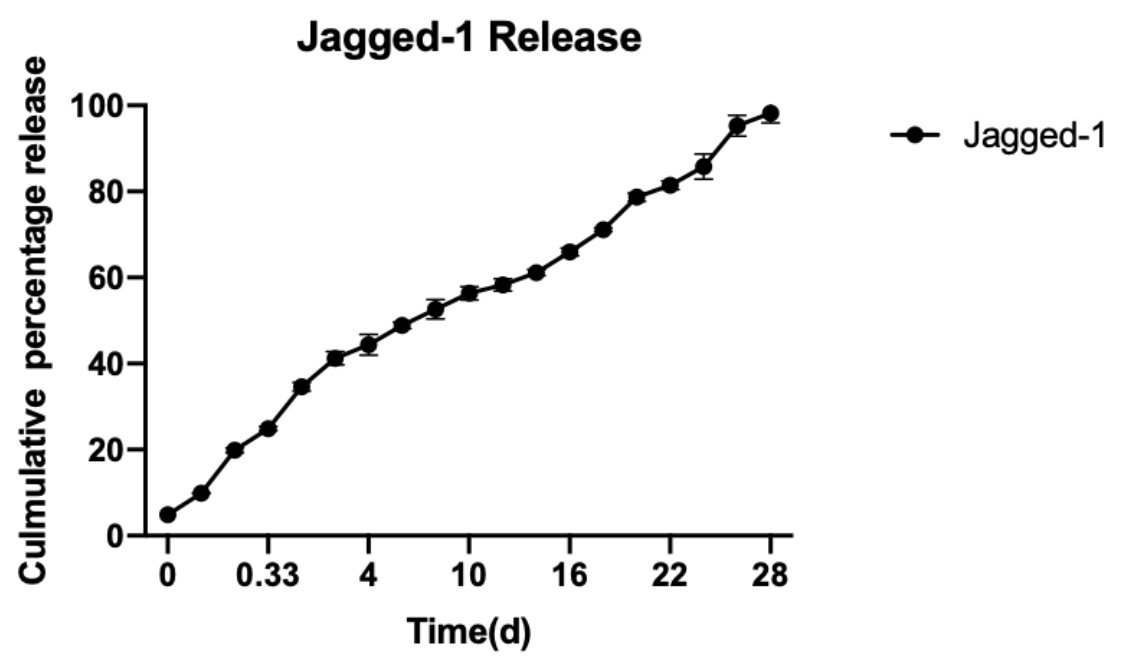

Figure S3: Jagged-1 peptide release from the hydrogel. Kinetics of Jagged-1 release from the hydrogel $(6.3 \mathrm{mg} / \mathrm{mL}$ Jagged-1 peptide loaded hydrogels $(100 \mu$ l per hydrogel, $\mathrm{n}=4)$ were incubated with $1 \mathrm{~mL}$ PBS solution with $0.02 \mathrm{mg} / \mathrm{mL}$ hyaluronidase at $37^{\circ} \mathrm{C}$. 


\section{Young's modulus}

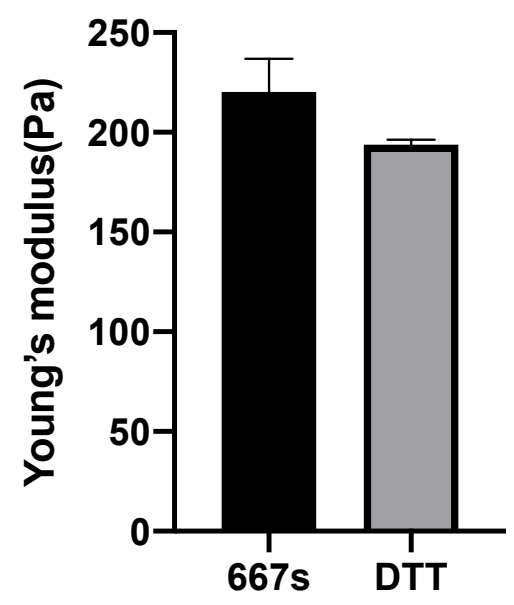

Figure S4: The Young's modulus of 2D hydrogels crosslinked by UV light and 2D hydrogels crosslinked by the DTT. 
A

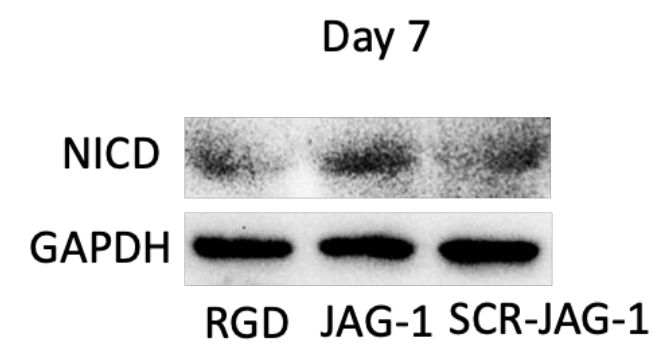

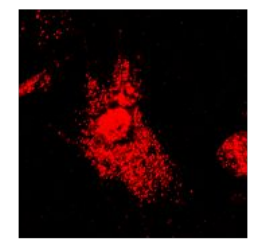

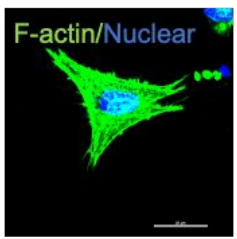

RGD

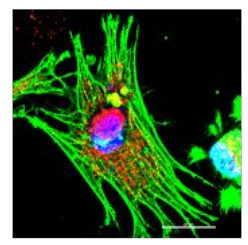

JAG-1
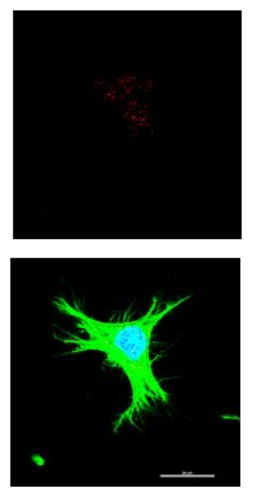

SCR-JAG-1
B

NICD

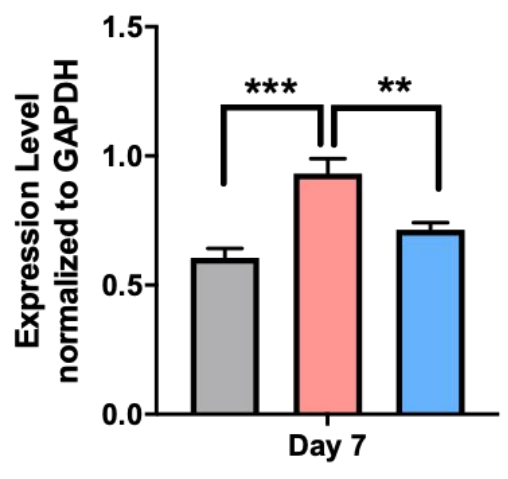

Time(d)

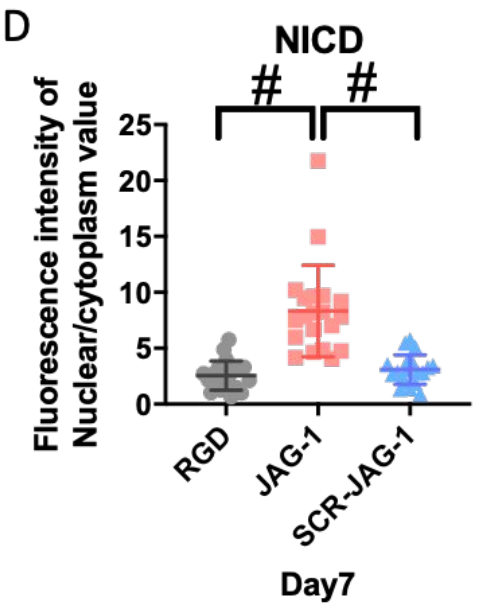

Figure S5: A) Western blot bands and B) quantification of the expression of NICD in each group. C) and D) Immunofluorescence staining and quantification of staining intensity for NICD in the hMSCs cultured on the peptide-functionalized hydrogels. (scale bar: $25 \mu \mathrm{m}$ ) Statistical significance: $* \mathrm{P}<0.05,{ }^{* *} \mathrm{P}<0.01,{ }^{* * *} \mathrm{P}<0.001$ and $\# \mathrm{P}<0.0001$. 

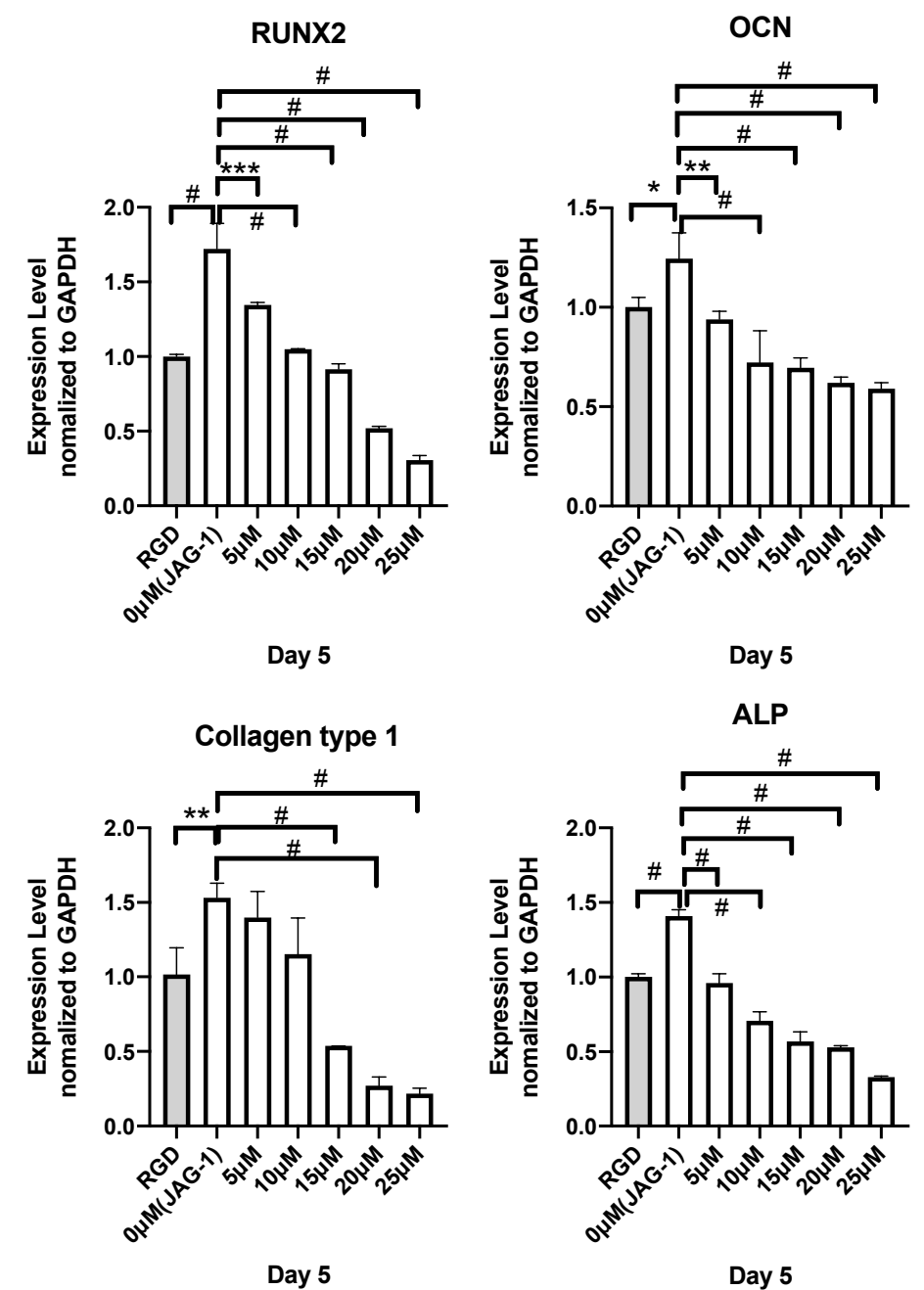

Figure S6: Quantitative gene expression results of osteogenic markers including RUNX2, OCN, COLI and ALP by the seeded hMSCs in porous hydrogels treated with various concentration of DAPT. Statistical significance: $* \mathrm{P}<0.05, * * \mathrm{P}<0.01, * * * \mathrm{P}<0.001$ and $\# \mathrm{P}<0.0001$. 
RGD

A

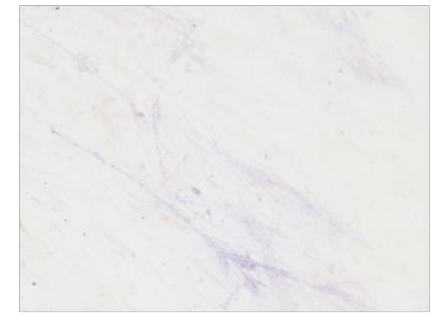

JAG-1

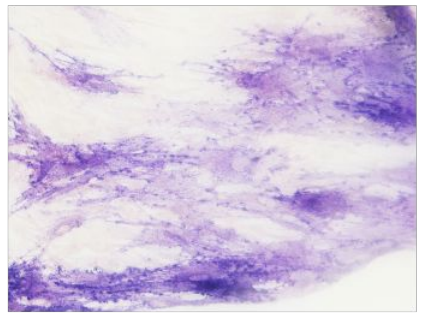

SCR-JAG-1

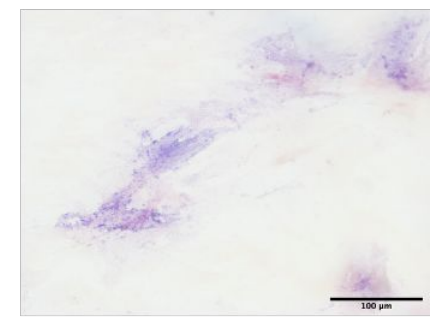

B

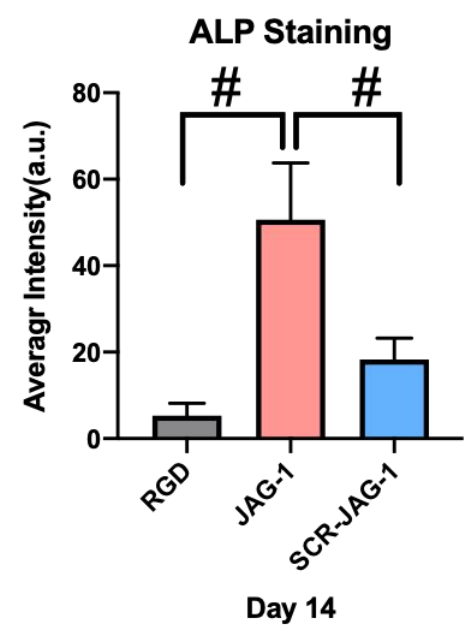

Figure S7: A) ALP staining results and B) quatification of the seeded hMSCs on 2D hydrogels cultured with osteogenic medium for 14 days $(\mathrm{n}=9)$. Statistical significance: $* \mathrm{P}<0.05, * * \mathrm{P}<$ $0.01, * * * \mathrm{P}<0.001$ and $\# \mathrm{P}<0.0001$. 


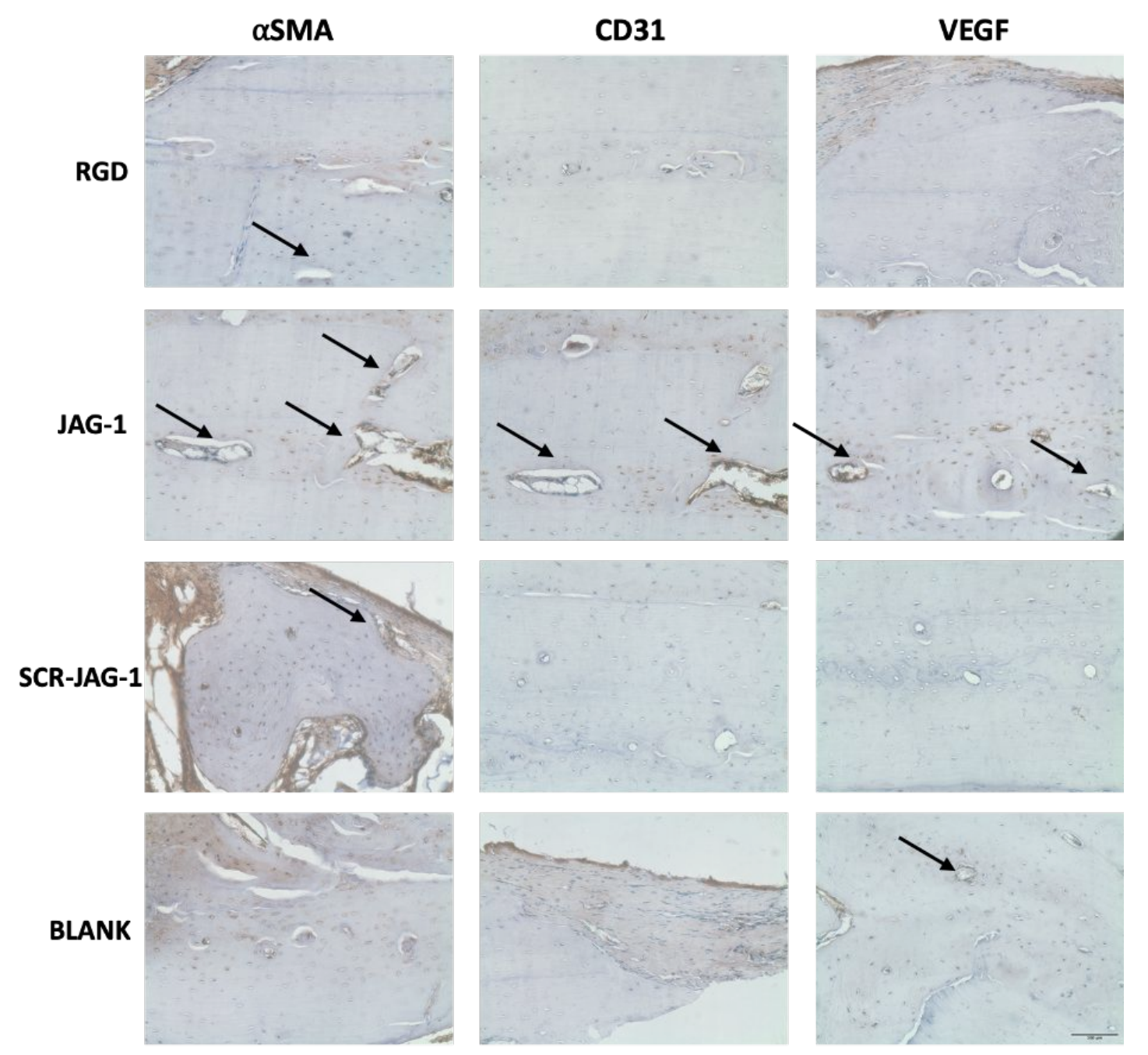

Figure S8: Immunohistochemical staining of angiogenic markers ( $\alpha$ SMA, CD31 and VEGF) in the RGD, JAG-1, SCR-JAG-1 groups and no hydrogels (Blank) after 8 weeks implantation $(n=4)$ 
A
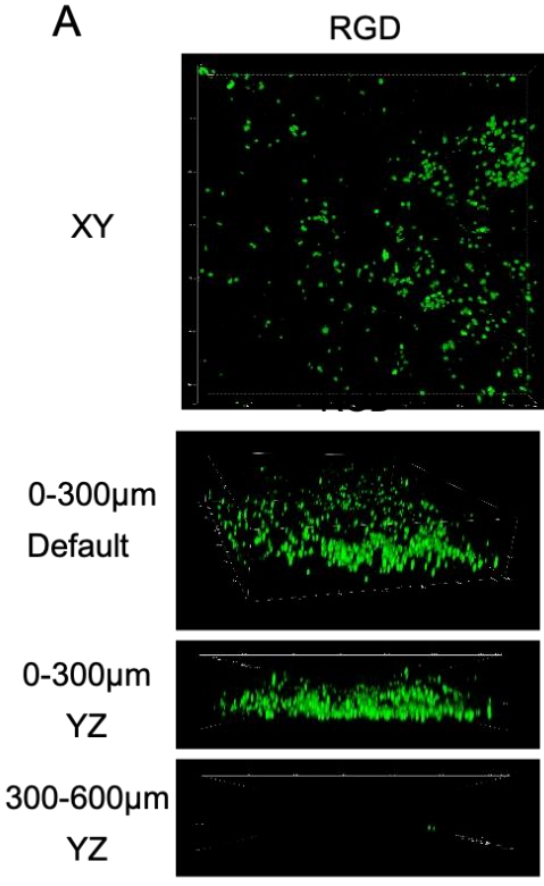

B

Fluorescence Intensity

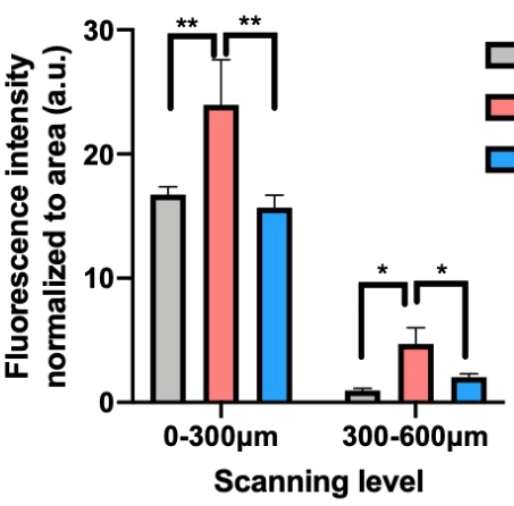

JAG-1
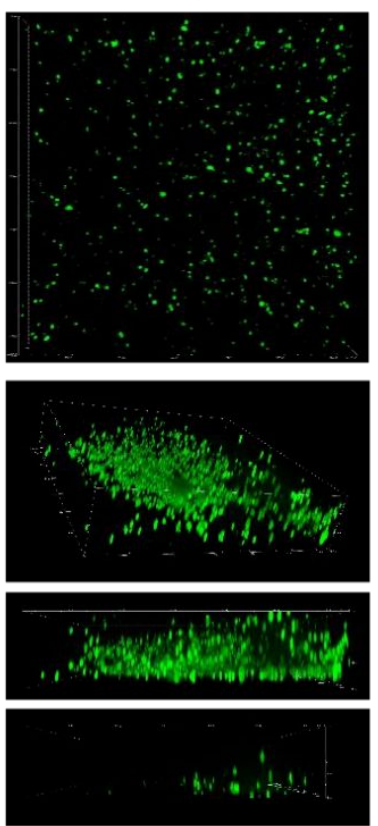

hit itis:
SCR-JAG-1
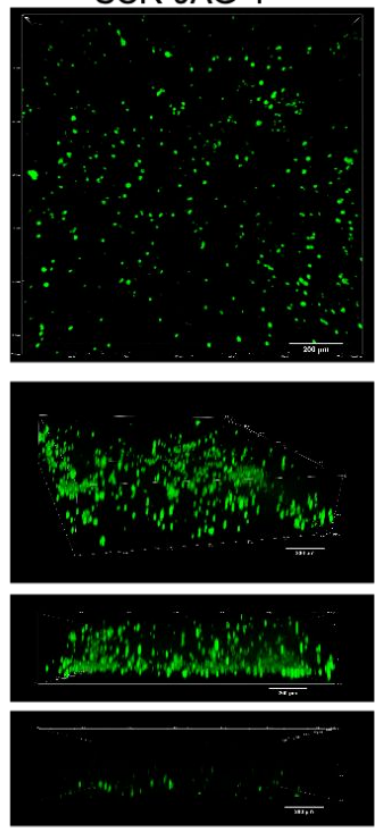

DNA

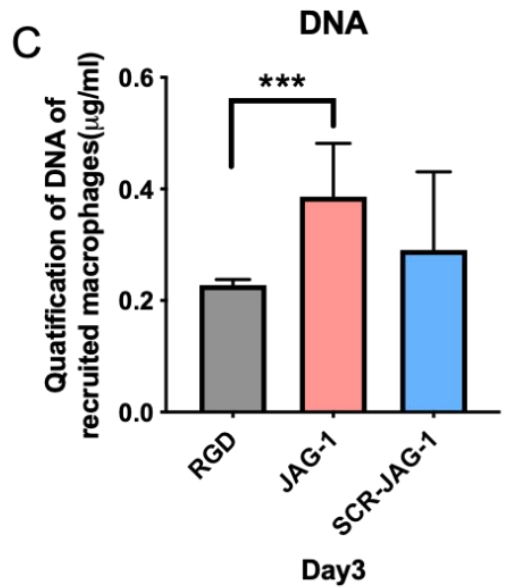

Figure S9: A) The live (green) and dead (red) staining and B) quatification of macrophages recruited by three groups of hydrogels in different scanning levels taken by confocal microscopy (Scale bar:200 $\mu \mathrm{m})$ C) Quantification of the DNA of macrophages recruited by three groups of hydrogels. Statistical significance: $* \mathrm{P}<0.05, * * \mathrm{P}<0.01, * * * \mathrm{P}<0.001$ and $\# \mathrm{P}<0.0001$. 


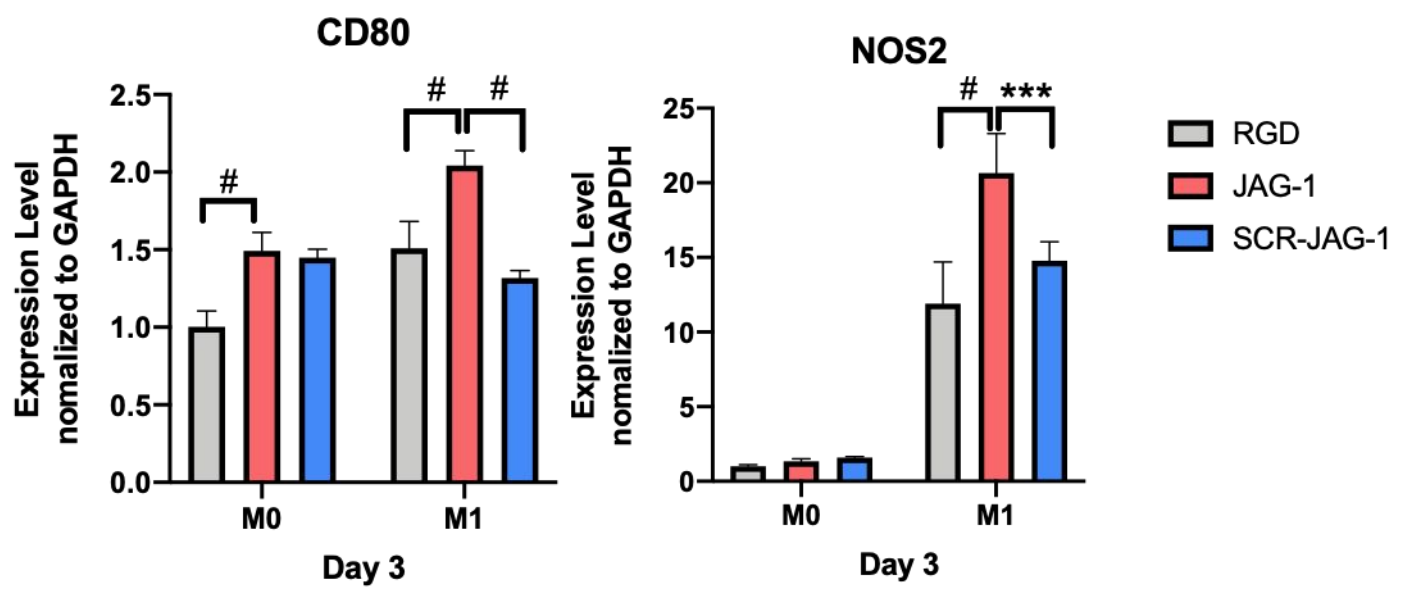

Figure S10 Quantitative gene expression results of M1 polarization markers including CD80 and NOS2 by the recruited macrophages in the macrophage basal medium. Statistical significance: *P $<0.05, * * \mathrm{P}<0.01, * * * \mathrm{P}<0.001$ and $\# \mathrm{P}<0.0001$.

\section{References:}

1. Nune, M.; Krishnan, U. M.; Sethuraman, S. Decoration of Plga Electrospun Nanofibers with Designer Self-Assembling Peptides: A "Nano-on-Nano" Concept. RSC Adv. 2015, 5(108), 88748-88757, 10.1039/C5RA13576A. DOI: 10.1039/C5RA13576A.

2. Oh, S.; Moon, K. S.; Lee, S. H. Effect of Rgd Peptide-Coated Tio Nanotubes on the Attachment, Proliferation, and Functionality of Bone-Related Cells. J. Nanomater. 2013, 2013, 965864. DOI: $10.1155 / 2013 / 965864$. 